\title{
Hippocampal Theta-Phase Modulation of Replay Correlates with Configural-Relational Short-Term Memory Performance
}

\author{
Claudia Poch, ${ }^{1}$ Lluis Fuentemilla, ${ }^{2,3}$ Gareth R. Barnes, ${ }^{4}$ and Emrah Düzel, ${ }^{3,5,6}$ \\ ${ }^{1}$ Laboratory of Cognitive and Computational Neuroscience, Centre for Biomedical Technology, Technical University of Madrid and Complutense University \\ of Madrid, 28223 Madrid, Spain, ${ }^{2}$ Institute of Biomedicine Research of Bellvitge (IDIBELL) Feixa Llarga s/n, 08907 L'Hospitalet (Barcelona), Spain, \\ ${ }^{3}$ Institute of Cognitive Neuroscience, University College London, WC1N 3AR London, United Kingdom, ${ }^{4}$ Wellcome Trust Centre for Neuroimaging, University \\ College London, WC1N 3BG London, United Kingdom, ${ }^{5}$ Institute of Cognitive Neurology and Dementia Research, Otto von Guericke University, 39120 Magdeburg, \\ Germany, and ${ }^{6}$ German Centre for Neurodegenerative Disorders-Magdeburg, Otto von Guericke University, 39120 Magdeburg, Germany
}

There is now growing evidence that the hippocampus generates theta rhythms that can phase bias fast neural oscillations in the neocortex, allowing coordination of widespread fast oscillatory populations outside limbic areas. A recent magnetoencephalographic study showed that maintenance of configural-relational scene information in a delayed match-to-sample (DMS) task was associated with replay of that information during the delay period. The periodicity of the replay was coordinated by the phase of the ongoing theta rhythm, and the degree of theta coordination during the delay period was positively correlated with DMS performance. Here, we reanalyzed these data to investigate which brain regions were involved in generating the theta oscillations that coordinated the periodic replay of configuralrelational information. We used a beamformer algorithm to produce estimates of regional theta rhythms and constructed volumetric images of the phase-locking between the local theta cycle and the instances of replay (in the $13-80 \mathrm{~Hz}$ band). We found that individual differences in DMS performance for configural-relational associations were related to the degree of phase coupling of instances of cortical reactivations to theta oscillations generated in the right posterior hippocampus and the right inferior frontal gyrus. This demonstrates that the timing of memory reactivations in humans is biased toward hippocampal theta phase.

\section{Introduction}

The process of maintaining information in the absence of external stimulation relies on the continuing firing of content-specific neuronal populations (Fuster and Alexander, 1971). There is now converging evidence from different cognitive domains that neural firing can be functionally related to the oscillatory dynamics of neural assembly behavior (O'Keefe and Recce, 1993; H. Lee et al., 2005; Jacobs et al., 2007; Rutishauser et al., 2010) and data from nonhuman primates indicate that such oscillatory coordination of neural firing also plays a functional role in memory maintenance (H. Lee et al., 2005; Siegel et al., 2009). According to an influential computational model (Lisman and Idiart, 1995; Jensen and Lisman, 1998; Lisman, 2010), maintenance is enabled by a cross-frequency coupling of gamma- (30-80 Hz oscillations) related neural spiking to specific phases of ongoing hippocampal

Received Dec. 3, 2010; revised March 16, 2011; accepted March 23, 2011.

Author contributions: L.F. and E.D. designed research; L.F. and E.D. performed research;C.P., L.F., G.R.B., and E.D. analyzed data; C.P., L.F., G.R.B., and E.D. wrote the paper.

This work was supported by the Spanish Government (Grants AP2009-4131 to C.P and RYC-2009-05471 to L.F.) and the Deutsche Forschungsgemeinschaft (SFB 776/A7 to E.D.). The Wellcome Trust Centre for Neuroimaging is supported by a strategic award from the Wellcome Trust.

The authors declare no competing financial interests.

Correspondence should be addressed to Emrah Düzel, Institute of Cognitive Neuroscience, University College London, WC1N 3AR London, UK. E-mail: e.duzel@ucl.ac.uk.

DOI:10.1523/JNEUROSCI.6305-10.2011

Copyright $\odot 2011$ the authors $\quad 0270-6474 / 11 / 317038-05 \$ 15.00 / 0$ theta oscillations $(4-8 \mathrm{~Hz})$. In agreement with this model, theta/ gamma coupling has been observed in relation to sequential (Axmacher et al., 2010) and spatial (Tort et al., 2009) shortterm memory.

Fuentemilla et al. (2010) investigated theta-coupled periodic replay during short-term memory maintenance using wholehead magnetoencephalographic (MEG) recordings. They trained a multivariate pattern classification (MVPC) algorithm on highfrequency (beta and gamma frequency range) oscillatory responses during the encoding phase of a visual delay match-tosample (DMS) task. By applying the trained MVPC algorithm to the delay period, they were able to detect instances of replay during the entire maintenance interval. The amount of replay was larger if the DMS task required that configural-relational information about the sample images (object-location associations within photographs of natural scenes) was maintained compared with a nonconfigural version of the DMS task. The periodicity of the replay was coordinated by the phase of the ongoing theta rhythm and the degree of theta coordination during the maintenance period was positively correlated with performance in the configural-relational DMS condition.

Although this study highlighted the behavioral significance of cross-frequency coupling for the maintenance of configural-relational information, it left open which brain regions generated the theta oscillations and which phase biased beta/gamma oscillations. There is growing evidence that the hippocampus can 
generate theta rhythms during maintenance that can be coherent with distant neocortical activity (Hyman et al., 2005; Jones and Wilson, 2005; Siapas et al., 2005; Benchenane et al., 2010) and can phase-bias fast oscillating neural populations outside limbic areas (Sirota et al., 2008).

In the current study, we explored which brain regions were involved in generating the theta oscillations that coordinated the periodic replay of configural-relational information in the study of Fuentemilla et al. (2010). We predicted that a hippocampal theta generator would phase couple to instances of replay in the delay interval of configural-relational maintenance and that interindividual variability in the degree of phase coupling of the hippocampal theta generator would correlate with memory performance.

\section{Materials and Methods}

The data were taken from Fuentemilla et al. (2010). Hence, information about participants, stimuli, task, data acquisition, and multivariate pattern classification analysis is as in Fuentemilla et al. (2010). These aspects of the methods will be briefly summarized here.

Participants. Eight right-handed healthy subjects (six males; mean age, 21 years; SD, 1.3 years) participated in the experiment after giving written informed consent (Fuentemilla et al., 2010). The study was approved by the University College London Research Ethics Committee for humanbased research. Subjects were financially compensated for participation.

Stimuli and task. The stimuli were photographs of indoor and outdoor scenes. The experiment consisted of two types of tasks: a DMS and a control task. The DMS task had a nonconfigural and a configural-relational condition. In all three conditions, the trial structure and stimulustiming were identical. After a $2 \mathrm{~s}$ intertrial interval, an indoor or an outdoor scene (sample) was presented for $3 \mathrm{~s}$. This was followed by a blank screen with a fixation cross for $5 \mathrm{~s}$ (delay period) and then by two test stimuli (probes) for $5 \mathrm{~s}$.

Retention of the sample stimulus across the $5 \mathrm{~s}$ delay period was necessary only in the two DMS conditions. Here, the two test images after the delay interval were used to probe memory for the sample. In the nonconfigural DMS condition, one of the probes was an exact repetition of the sample and the other depicted an entirely different novel scene of the same category (indoor or outdoor) as the sample. In the configuralrelational DMS condition, the nonmatching probe stimuli were very similar to the sample. In the control task, the probe images were always different from the sample and subjects were instructed to indicate whether the probes were identical or different (without maintaining the sample). In half of the trials, the probes were identical and in the other half, they differed to the same degree as the probes in the configuralrelational DMS condition.

Configural-relational, nonconfigural, and control conditions were separated into blocks of 10 trials each. Subjects were instructed before each block as to which task condition would be tested. There were four blocks, resulting in 40 trials per task condition. The presentation of indoor/outdoor stimuli was counterbalanced across each block and was kept constant across individual trials.

Data acquisition. MEG data $(480 \mathrm{~Hz}$ sampling rate, $120 \mathrm{~Hz}$ low-pass filtering) were recorded using a 275-channel CTF Omega whole-head gradiometer (VSM MedTech). Head movements during the recording sessions were monitored with localizer coils at the nasion and $1 \mathrm{~cm}$ anterior of the left and right tragus.

MEG multivariate pattern classification MVPC analysis. To test whether category-selective patterns of activity elicited during sensory input would be reactivated during the delay interval, Fuentemilla et al. (2010) used a MVPC algorithm. MVPCs were trained on frequency features computed by a time-frequency (TF) analysis. Epochs of $9 \mathrm{~s}$, including a $1000 \mathrm{~ms}$ baseline preceding the onset of sample presentation, 3000 $\mathrm{ms}$ of sample presentation, and $5000 \mathrm{~ms}$ of maintenance period, were used in the TF analysis. Data were downsampled to $250 \mathrm{~Hz}$ before TF analysis. TF was computed by a continuous wavelet transformation on single-trial data for each subject and sensor using a complex Morlet with the relation $f_{0} / \sigma_{f}$ set to 7 .
The instantaneous power at 48 frequencies, spanning a range from 13 to $79 \mathrm{~Hz}$ every $80 \mathrm{~ms}$, and for each single sensor were used to train MVPCs. Note that low-frequency components $(2-12 \mathrm{~Hz})$ were not used to train MVPCs.

A different classifier was trained for each of 11 time points spanning a period from $36 \mathrm{~ms}$ before to $764 \mathrm{~ms}$ after sample onset $(-36,44,124$, $204,284,364,444,524,604,684$, and $764 \mathrm{~ms}$ relative to the sample onset). Each of the 11 classifiers was trained with data from each experimental condition separately. This yielded 40 training patterns ( 20 per category) for each of the 11 classifiers and for each experimental condition (control, nonconfigural, and configural-relational). Here, neural network optimization (i.e., learning) was based on the conjugate gradient algorithm (traincgb; Matlab) (Bishop, 1995). The target patterns were [1 $0]$ for an indoor scene and [0 1] for an outdoor scene.

First, the category-specific (indoor/outdoor scenes) neuronal representations that developed during the encoding period were determined. This was implemented using a cross-validation process (for details, see Fuentemilla et al., 2010). Second, the trained classifiers were used to test, at single-trial level, whether TF data of the maintenance interval could be accurately classified as indoor versus outdoor. For a given trained classifier, at 225 consecutive time points of the maintenance interval (corresponding to $4.5 \mathrm{~s}$ after excluding the first and the last $250 \mathrm{~ms}$ of the maintenance interval), they tested whether the trained classifiers could discriminate between indoor/outdoor scene maintenance based on selected TF features at that time point. At each time point on each trial, the classifier outputs were then thresholded [using a value of $>0.95$ from a possible output value range of 0 to 1 (perfect discrimination)]. The resulting thresholded output (for each of the 11 classifiers) was then set to $y=1$ for a correct output and $y=0$ for an incorrect output.

As outlined in Fuentemilla et al. (2010), reactivations were defined operationally as patterns producing correct classifier outputs for any of the 225 time points of the delay interval. To identify reactivations in each condition, classification accuracy of delay activity was computed separately for each category (indoor/outdoor). If $C$ is the number of trials correctly classified then $P(C)$ follows a binomial distribution with correct probability $r$ and $n=20$ (20 observations of each trial type). Testing against the null hypotheses of classification at the chance level $(r=0.5)$ using the normal approximation to the binomial density allowed us to compute $p$ values. A Bonferroni correction for multiple comparison was then applied to give a test-wise threshold of $p=1.8 \times 10^{-5}$ (i.e., 225 time points $\times 11$ classifiers) for a family-wise error rate of 0.05 . This corresponded to 20/20 correct trials (i.e., perfect classification accuracy). Reactivation times were defined as those time points for which the classification accuracy reached this threshold.

Source reconstruction. The linearly constrained minimum variance scalar beamformer spatial filter algorithm (Sekihara et al., 2004) implemented in SPM8 was used to generate maps of source activity in a $10 \mathrm{~mm}$ grid. Coregistration to the MNI coordinates was based on three fiducials points: nasion and left and right preauricular. The forward model was based on the single-shell model fit to inner skull surface of the inverse normalized SPM template. The beamformer source reconstruction is based on two stages. First, calculation of the weights that map sensors to source locations (constructed from the covariance window). Second, multiplication of raw data by these weights to obtain a time-series estimate at each source location. In this case, the time varying estimate of the electromagnetic activity at each of the locations of the image was projected through the spatial filter constructed from the covariance matrix comprising $5 \mathrm{~s}$ (maintenance period) of the three conditions in the frequency range of $4-8 \mathrm{~Hz}$.

Theta phase locking to reactivations times. The local time-series estimates were bandpass filtered with a zero phase filter, with a $f_{0}$ of $6 \mathrm{~Hz}$ and bandwidth of $\pm 1 \mathrm{~Hz}$ ( 0.48 octaves). A frequency of $6 \mathrm{~Hz}$ was chosen on the basis of data from Fuentemilla et al. (2010) showing significant phase coupling of reactivation times to $6 \mathrm{~Hz}$ theta oscillations. The instantaneous phase complex representation of the filtered signal was calculated as follows: $e^{i \varphi(t)}=s_{\mathrm{a}}(t) /\left|s_{\mathrm{a}}(t)\right|$, where $s_{\mathrm{a}}(t)$ is the signal's analytic representation. Phase coupling of reactivations to a certain theta oscillation was calculated as the phase alignment of the phases given by the reacti- 
vations vector. For each subject, this phase alignment was measured with the phase locking value (PLV) of Tallon-Baudry et al. (1996), as follows:

$$
\operatorname{PLV}_{j}=\frac{1}{N_{j}} \cdot \frac{1}{M}\left|\sum_{n=1}^{N_{j}} \sum_{m=1}^{M} \exp \left(i \phi_{m n j}\right)\right|,
$$

where $N_{j}$ represents the number of reactivations for classifier $j$. And $\Phi_{n j}$ is the relative phase of the cortical theta signal relative to reactivation $n$, in trial $m$. That is, if all reactivations occur at random phases (distributed around the unit circle), then average magnitude of the PLV vector will tend toward zero, whereas if all reactivations occur at the same theta phase, the PLV will tend toward unity. PLV was calculated separately for each of the 10 classifiers trained at different time points (the classifier data from baseline was not included), and then averaged for each of the three task conditions.

To correct the PLV variability among subjects, a relative PLV was calculated as the ratio of the observed PLV to the empirically determined $p<0.005$ threshold. The empirical distribution of PLV was obtained by shifting randomly the phases of each of the trials by $\theta_{j}$ (drawn from a uniform distribution on $\{-\Pi, \Pi\})$, calculating the PLV as described above, and repeating these two steps 1000 times. A relative PLV $>1$ means that the real PLV is significant respect to the empirical distribution, where a relative PLV $<1$ means that the real PLV could be obtained by chance $(p>0.005)$.

Statistical analysis. The relative PLV maps were converted to NIFTI images to proceed with the statistical analysis in SPM8. A linear regression was conducted between the DMS behavioral performance vector (across subjects) and each of the source space PLV estimates in all three conditions. As we only expected to identify the theta coordinators of the replay that are functionally relevant for maintaining configural-relational associations, the control and nonconfigural conditions served as controls.

\section{Results}

The accuracy in identifying the matching sample in the configural-relational task was variable across subjects (mean, 79\%; SD, 13.3 ) and nearly perfect (mean, 97\%; SDm 0.02) in the nonconfigural task

To determine which brain regions showed theta phase coupling to reactivations varying as a function of performance in the configural-relational condition, a simple regression was conducted between the relative PLV and DMS performance. The test revealed a significant positive linear effect in two areas in the right hemisphere. A local maxima peak was found in the right posterior hippocampus $\left(30,-38,4 ; r=0.9, t_{(6)}=5.25, p<0.001\right.$, small volume corrected $p<0.05)$ and in the right inferior frontal gyrus (IFG; 54, 14, 12; $r=0.96, t_{(6)}=8.58, p<0.001$, small volume corrected $p<0.05$ ) (Fig. $1 B$ ).

Furthermore, the within-subjects permutation test also showed that almost every subject had a significant PLV in the two areas identified by the correlation analysis (Fig. $1 B$ ). The chance probability of six of eight subjects having a significant $(p<0.05)$ phase locking of reactivations to theta oscillations generated at these locations is extremely low $\left(p<6 \times 10^{-9}\right.$, binomial distribution for six of eight subjects reaching $p=0.05$ ). To exclude the possibility that these effects were due to signal/noise differences among subjects, a simple regression test between the performance accuracy in the configural-relational conditions and the nonconfigural-relational and control PLVs images was also conducted. These analyses did not yield any significant results (all $p>0.05)$.

These results show that performance accuracy in the configural-relational DMS condition is positively related to the amount of phase concentration of reactivation times to theta signals generated in the right posterior hippocampus and the right inferior frontal lobe. That is, higher PLV with theta signals generated in these two regions is associated with more accurate memory for the configural-relational probe.

\section{Discussion}

Individual differences in working memory performance for configural-relational associations were related to the degree of phase coupling of instances of cortical reactivations to theta oscillations generated in the right posterior hippocampus and the right inferior frontal gyrus. This demonstrates that the timing of memory reactivations in humans is biased toward hippocampal theta phase.

We classified reactivations on the basis of oscillatory frequencies comprising the beta and gamma frequency range. Therefore, the performance-related phase-coupling of reactivations to hippocampal and prefrontal theta rhythms points toward a cognitive role of cross-frequency phase-amplitude coupling between frontolimbic theta oscillations and neocortical beta and gamma oscillations.

It is likely that the configural-relational information represented in the natural images of scenes that we used in the current experiment is represented in a distributed cortical network that involves parietal, visual, inferior temporal, and frontal regions (Burgess, 2008). Thus, although we currently cannot determine the neural sources of beta and gamma oscillations that contributed to the classifiers' ability to decode reactivation, it is safe to assume that the corresponding networks were distributed and variable across individuals. Hence, our finding that behavioral performance in this task was correlated with phase coupling only to theta in right posterior hippocampus and right inferior frontal gyrus does not rule out widespread cortical entrainment. Instead, it is compatible with the possibility that neocortical generators of the relevant theta entrainments of beta and gamma oscillations may have been variable across participants in source space, whereas the hippocampal and right inferior frontal sources showed much less interindividual variability.

Cross-frequency coupling supporting maintenance of information is a hallmark of physiological models of maintaining sequential information in working memory (Lisman and Idiart, 1995; Lisman, 2010). Evidence that cross-frequency coupling of oscillations mediates a phase-dependent coding of items during maintenance in right frontal regions comes from a recent study in nonhuman primates (Siegel et al., 2009). In this study, maintaining both the identity and the order of two objects over a delay of $1 \mathrm{~s}$ was associated with prefrontal spike-local field potential (LFP) synchronization at $32 \mathrm{~Hz}$. This synchronization improved object decoding compared with spike rate alone. Also, maximal information in spikes about the identity of the first presented object was found in an earlier phase of the $32 \mathrm{~Hz}$ LFP cycle than information about the second object. This was accompanied by a significant modulation of the amplitude of $32 \mathrm{~Hz}$ oscillations by the phase of delta $(3 \mathrm{~Hz})$ oscillations. This cross-frequency interaction may be not only involved in sequential information processing but also in object-space representations, as it has been described in the rat's hippocampus (Tort, 1999).

Our findings are compatible with data from animal studies indicating that hippocampal theta oscillations can coordinate neural activity in distant brain regions during spatial memory tasks (Jones and Wilson, 2005; Sirota et al., 2008). Specifically, several experiments in rodents have demonstrated a phase locking of medial prefrontal activity to the hippocampal theta rhythm and this phase locking could be observed at the level of medial prefrontal neuronal spikes as well as LFPs (Jones and Wilson, 
A

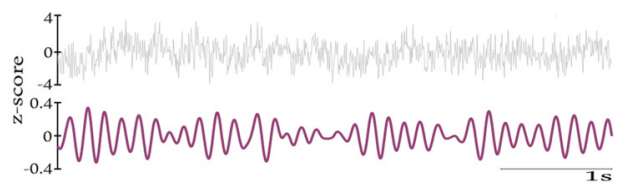

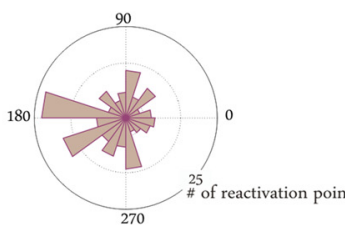

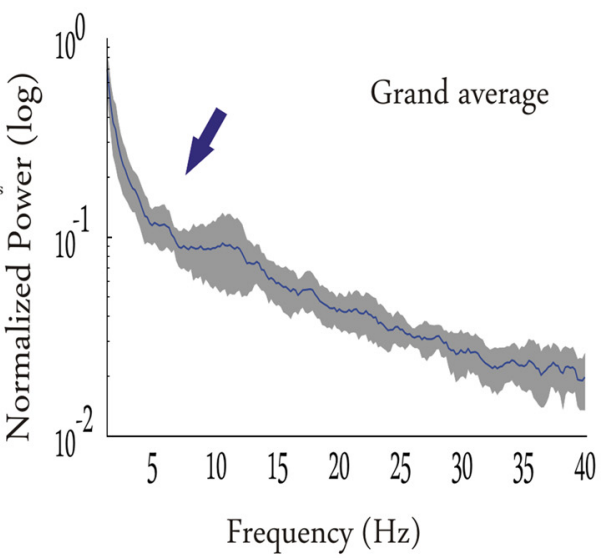

Right Hippocampus

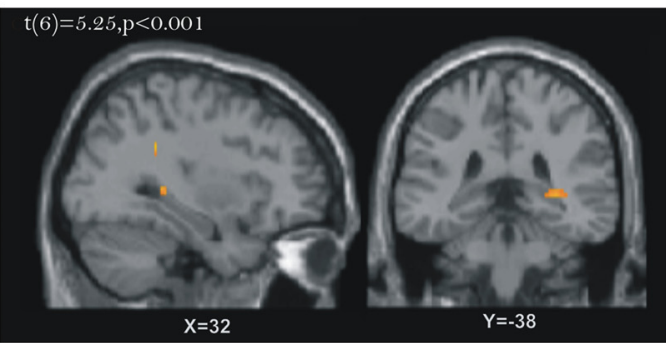

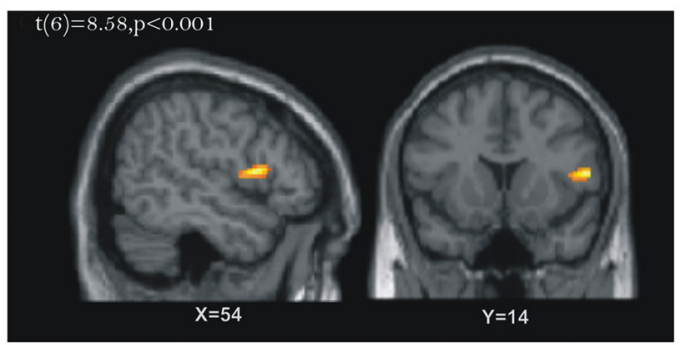
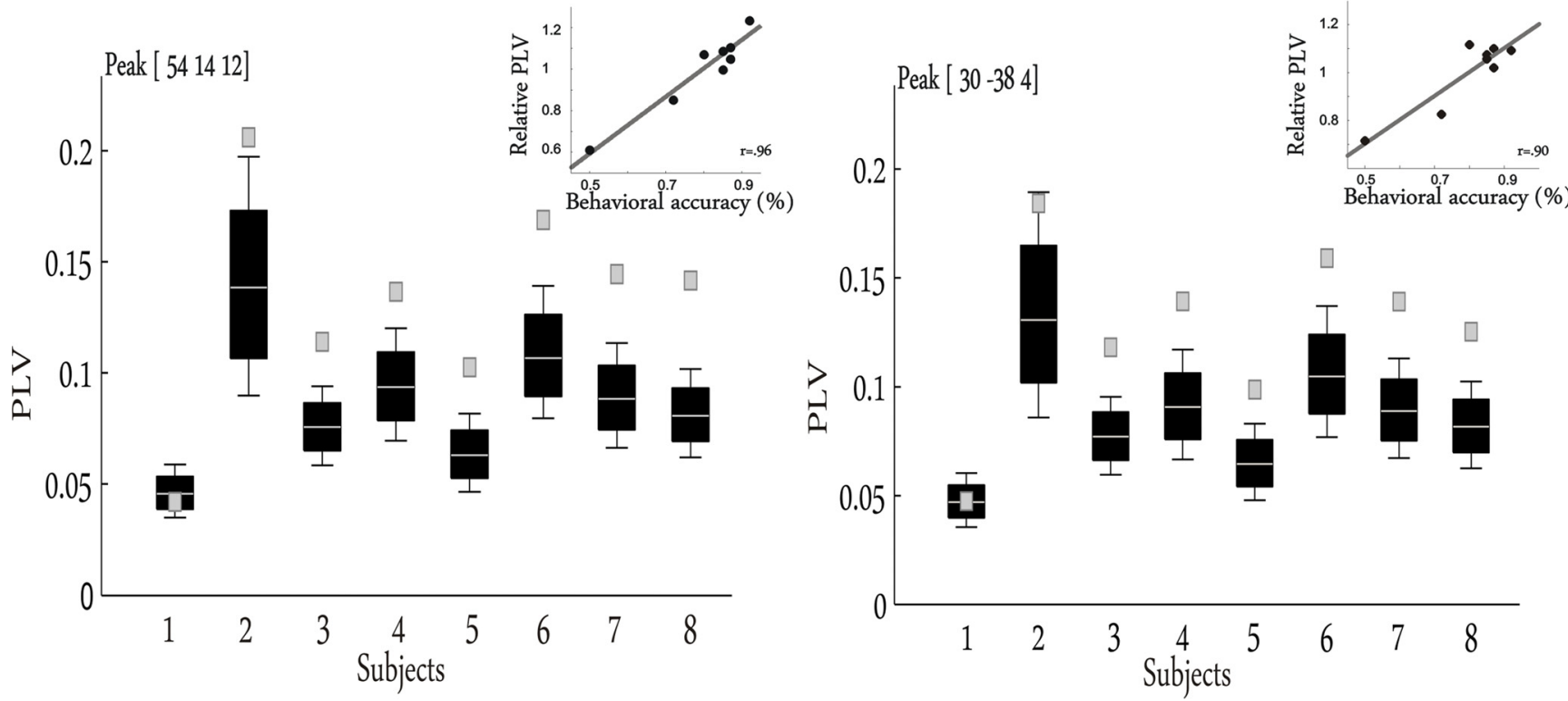

Figure 1. A, Illustration of hippocampal theta oscillations in the delay period of the configural-relational DMS condition. Left, Single trial of spatially (beamformer) filtered data from the right posterior hippocampus (bottom, bandpass filtered 5-7 Hz). Middle, Phase histogram of the preferred phases of the hippocampal theta at reactivation times (length of the radius shows the number of reactivations at a given phase across all trials; maximum $n=180$ ). Right, Population grand average Fourier spectrum from the right hippocampus (arrow, theta power peak at $\sim 6 \mathrm{~Hz}$; gray, SD). $B$, Brain regions (left, right inferior frontal gyrus; right, right posterior hippocampus) showing theta phase coupling to reactivations during the DMS delay varying as a function of performance. Statistical maps on the left are thresholded at $p<0.005$ and overlaid on a single subject T1-weighted MRI scan. Boxplots on the bottom depict the permutation distribution of phase coupling between theta oscillations and reactivations for each participant. The top and the bottom of the box represent one SD. The band near the middle of the box represents the median. The top whisker is the 95th value of the distribution. The gray square represents the real PLV, so a square above the top whisker indicates a significant $\operatorname{PLV}$ ( $p<0.05$, corrected). Inside the boxplots, Correlations of relative PLVs with memory performance (note that these correlations are shown for illustrative purposes only and no statistical inference was made based on these correlation plots).

2005; Siapas et al., 2005; Benchenane et al., 2010). The functional relevance of this theta network activity has been demonstrated in studies showing positive correlation between theta coherence and performance (Hyman et al., 2005; Benchenane et al., 2010) or task demands (Siapas et al., 2005).
It is interesting to note that cross-species comparisons suggest a correspondence of the rat medial prefrontal cortex (PFC) to the primate dorsolateral PFC (Uylings et al., 2003). In these comparisons, the definition of dorsolateral comprises also the inferior (or ventral) lateral aspect of the PFC (Uylings et al., 2003), a 
region that was implicated in our results as a theta generator showing significant PLV to replay. In fact, both the medial PFC in rats and the dorsolateral PFC in humans have been implicated in working memory performance and the inferior frontal region identified in our study has been shown to be involved in a variety of working memory tasks (Makuuchi et al., 2009). Hence, it can be speculated that the common hippocampal and inferior frontal theta phase coupling to replay in humans is functionally related to rodent studies showing coordinated hippocampal and medial PFC theta during maintenance demands (Jones and Wilson, 2005; Siapas et al., 2005; Benchenane et al., 2010).

There are several mechanisms through which hippocampally generated theta oscillations could phase bias neocortical activity. Hippocampal activity could entrain other pacemakers such as cholinergic populations in the basal forebrain (Petsche et al., 1962; Tóth et al., 1993; M. G. Lee et al., 2005) or the supramammillary nucleus (Kocsis and Vertes, 1994). Alternatively, the hippocampus (subfield CA1) could synchronize with monosynaptic targets in the entorhinal cortex (Colgin and Moser, 2006) and indirectly use their widespread connectivity. These two mechanisms could exploit the intrinsic pacemaker properties of neocortical neuronal subgroups (Blatow et al., 2003). Finally, there are sparse long-range hippocampal projections to distant neocortical regions (Jinno et al., 2007) as well as long-range GABAergic interneuron projections (e.g., to the retrosplenial and medial PFC cortex) (Jinno et al., 2007; Klausberger and Somogyi, 2008) that could directly entrain neocortical populations.

To summarize, we showed that it is possible to combine MVPC-based decoding of periodic replay in the beta and gamma frequency range with source analysis of the hippocampal slow oscillations that bias the timing and periodicity of replay. More generally, this approach offers a new perspective for mechanistic investigations of human memory processes. It helps to narrow an important gap that exists between noninvasive research in humans and invasive research in nonhuman animals, namely, the ability to access representation of memory content to be able to understand the mechanisms that enable its reactivation.

\section{References}

Axmacher N, Henseler MM, Jensen O, Weinreich I, Elger CE, Fell J (2010) Cross-frequency coupling supports multi-item working memory in the human hippocampus. Proc Natl Acad Sci U S A 107:3228-3233.

Benchenane K, Peyrache A, Khamassi M, Tierney PL, Gioanni Y, Battaglia FP, Wiener SI (2010) Coherent theta oscillations and reorganization of spike timing in the hippocampal-prefrontal network upon learning. Neuron 66:921-936.

Bishop CM (1995) Neural networks for pattern recognition. New York: Oxford UP.

Blatow M, Rozov A, Katona I, Hormuzdi SG, Meyer AH, Whittington MA, Caputi A, Monyer H (2003) A novel network of multipolar bursting interneurons generates theta frequency oscillations in neocortex. Neuron $38: 805-817$

Burgess N (2008) Spatial cognition and the brain. Ann N Y Acad Sci 1124:77-97.

Colgin LL, Moser EI (2006) Neuroscience: rewinding the memory record. Nature 440:615-617.

Fuentemilla L, Penny WD, Cashdollar N, Bunzeck N, Duzel E (2010) Thetacoupled periodic replay in working memory. Curr Biol 20:606-612.
Fuster JM, Alexander GE (1971) Neuron activity related to short-term memory. Science 173:652-654.

Hyman JM, Zilli EA, Paley AM, Hasselmo ME (2005) Medial prefrontal cortex cells show dynamic modulation with the hippocampal theta rhythm dependent on behavior. Hippocampus 15:739-749.

Jacobs J, Kahana MJ, Ekstrom AD, Fried I (2007) Brain oscillations control timing of single-neuron activity in humans. J Neurosci 27:3839-3844.

Jensen O, Lisman JE (1998) An oscillatory short-term memory buffer model can account for data on the Sternberg task. J Neurosci 18:10688-10699.

Jinno S, Klausberger T, Marton LF, Dalezios Y, Roberts JD, Fuentealba P, Bushong EA, Henze D, Buzsáki G, Somogyi P (2007) Neuronal diversity in GABAergic long-range projections from the hippocampus. J Neurosci 27:8790-8804

Jones MW, Wilson MA (2005) Theta rhythms coordinate hippocampalprefrontal interactions in a spatial memory task. PLoS Biol 3:e402.

Klausberger T, Somogyi P (2008) Neuronal diversity and temporal dynamics: the unity of hippocampal circuit operations. Science 321:53-57.

Kocsis B, Vertes RP (1994) Characterization of neurons of the supramammillary nucleus and mammillary body that discharge rhythmically with the hippocampal theta rhythm in the rat. J Neurosci 14:7040-7052.

Lee H, Simpson GV, Logothetis NK, Rainer G (2005) Phase locking of single neuron activity to theta oscillations during working memory in monkey extrastriate visual cortex. Neuron 45:147-156.

Lee MG, Hassani OK, Alonso A, Jones BE (2005) Cholinergic basal forebrain neurons burst with theta during waking and paradoxical sleep. J Neurosci 25:4365-4369.

Lisman J (2010) Working memory: the importance of theta and gamma oscillations. Curr Biol 20:R490-R492.

Lisman JE, Idiart MA (1995) Storage of $7 \pm 2$ short-term memories in oscillatory subcycles. Science 267:1512-1515.

Makuuchi M, Bahlmann J, Anwander A, Friederici AD (2009) Segregating the core computational faculty of human language from working memory. Proc Natl Acad Sci U S A 106:8362-8367.

O’Keefe J, Recce ML (1993) Phase relationship between hippocampal place units and the EEG theta rhythm. Hippocampus 3:317-330.

Petsche H, Stumpf C, Gogolak G (1962) The significance of the rabbit's septum as a relay station between the midbrain and the hippocampus. I. The control of hippocampus arousal activity by the septum cells. Electroencephalogr Clin Neurophysiol 14:202-211.

Rutishauser U, Ross IB, Mamelak AN, Schuman EM (2010) Human memory strength is predicted by theta-frequency phase-locking of single neurons. Nature 464:903-907.

Sekihara K, Nagarajan SS, Poeppel D, Marantz A (2004) Asymptotic SNR of scalar and vector minimum-variance beamformers for neuromagnetic source reconstruction. IEEE Trans Biomed Eng 51:1726-1734.

Siapas AG, Lubenov EV, Wilson MA (2005) Prefrontal phase locking to hippocampal theta oscillations. Neuron 46:141-151.

Siegel M, Warden MR, Miller EK (2009) Phase-dependent neuronal coding of objects in short-term memory. Proc Natl Acad Sci U S A 106:21341-21346.

Sirota A, Montgomery S, Fujisawa S, Isomura Y, Zugaro M, Buzsáki G (2008) Entrainment of neocortical neurons and gamma oscillations by the hippocampal theta rhythm. Neuron 60:683-697.

Tallon-Baudry C, Bertrand O, Delpuech C, Pernier J (1996) Stimulus specificity of phase-locked and non-phase-locked $40 \mathrm{~Hz}$ visual responses in human. J Neurosci 16:4240-4249.

Tort AB, Komorowski RW, Manns JR, Kopell NJ, Eichenbaum H (2009) Theta-gamma coupling increases during the learning of item-context associations. Proc Natl Acad Sci U S A 106:20942-20947.

Tóth K, Borhegyi Z, Freund TF (1993) Postsynaptic targets of GABAergic hippocampal neurons in the medial septum-diagonal band of broca complex. J Neurosci 13:3712-3724.

Uylings HB, Groenewegen HJ, Kolb B (2003) Do rats have a prefrontal cortex? Behav Brain Res 146:3-17. 